\title{
Double drainage of total anomalous pulmonary venous connection revealed after surgical repair of a supracardiac total anomalous pulmonary venous connection
}

\author{
Hiroyuki Nagao ${ }^{1}$, Kenta Tominaga ${ }^{2}$, Naoya Kamei $^{2}$, and Toshikatsu Tanaka ${ }^{2}$ \\ ${ }^{1}$ National Cerebral and Cardiovascular Center \\ ${ }^{2}$ Hyogo Prefectural Kobe Children's Hospital
}

July 7, 2020

\begin{abstract}
Total anomalous pulmonary venous connection is an anomaly where the pulmonary veins are directly connected to one of the systemic veins or to the right atrium. The most common classification of this condition is by Craig, Darling and Rothney, which is based on the anatomic site of abnormal connection
\end{abstract}

Abstract

Total anomalous pulmonary venous connection (TAPVC) is an anomaly where the pulmonary veins are directly connected to one of the systemic veins or to the right atrium. The most common classification of this condition is by Craig, Darling and Rothney, which is based on the anatomic site of the abnormal connection. A few variants are reported which do not fit into these classic forms.

We report 2 cases that previously underwent surgical repair of supracardiac TAPVC, where the pulmonary veins drained into the innominate vein through an ascending vertical vein. However these cases did not fit into the classic forms, because in both cases, a second vertical vein was detected incidentally during a follow-up diagnostic catheterization 1 year after the initial surgery. The second veins originated proximal to the ligated vertical vein and drained into the superior vena cava through the accessory hemiazygos vein and azygos vein.

In Case 1, which was did not provoke right heart volume overload and general condition is good. the patient is followed up carefully, even in the absence of any intervention or surgical intervention.

In Case 2, we confirmed right heart volume overload, and then performed coil embolization of the veno-venous shunt (VV shunt) of the second vertical vein.

This report describes a successful intervention by catheterization in the treatment of a VV shunt of TAPVC.

\section{Introduction}

Total anomalous pulmonary venous connection (TAPVC) constitutes $1 \%-1.5 \%$ of congenital heart diseases in children. Four types have been identified based on where the pulmonary vein connects abnormally: supracardiac, cardiac, infracardiac, and mixed, where the anomalous connection occurs at 2 or more of the above levels. [1] [2]

In the 2 cases discussed in this report, a second vertical vein was noticed incidentally during routine follow-up catheterization 1 year after the initial surgery. 
It originated proximally from the ligated vertical vein and drained into the superior vena cava through the accessory hemiazygos vein.

It seemed that blood perfusion to a second vertical vein had increased due to disappear of dominant vertical vein blood perfusion after the initial surgery.

In this report we discuss the management of this rare case. Furthermore, this is the first reported case of a transcatheter closure of a second vertical vein in a mixed TAPVC.

\section{Case Report}

\section{Case 1}

A 9 year-old boy underwent surgical repair of supracardiac TAPVC when he was 2 months old and weighed 2.2 Kg. [Figure1]

During the operation, the vertical vein was ligated. One year after the surgical repair, we performed follow up diagnostic catheterization that showed obvious azygos vein enlargement. Contrast enhanced CT was performed, and it showed a veno-venous shunt (VV shunt) that originated proximal to the ligated vertical vein and drained into the superior vena cava through the accessary hemiazygos vein-azygos vein. [Figure2]

These findings showed there was a second vertical vein causing TAPVC. Therefore, we corrected the diagnosis from a supracardiac TAPVC to a double drainage of TAPVC, a rare variant of the mixed type. The leftright shunting, which occurred as a result of this development, did not provoke right heart volume overload, and the general condition of the patient was good. His right heart function is under close monitoring in outpatient.

\section{Case 2}

A 2 year-old infant, weighing $9 \mathrm{~kg}$, with a diagnosis of supracardiac TAPVC underwent surgical correction at 1 month. Preoperative echocardiography and contrast CT showed that the pulmonary veins formed a confluence and drained into the innominate vein through the vertical vein. We diagnosed supracardiac TAPVC without another cardiac anomaly.

During surgery, the vertical vein was ligated on the innominate vein side. Postoperatively, recovery was good and pulmonary venous stenosis (PVS) did not arise. Furthermore, using contrast CT, we confirmed that the connection of the common chamber and left atrium was not restrictive before the patient was discharged. A year after the surgical repair, we performed a follow up catheter examination to check the patients' cardiac condition. It showed that central venous pressure was $5 \mathrm{mmHg}$, right ventricular pressure was $23 \mathrm{mmHg}$, mean pulmonary capillary wedge pressure $9 \mathrm{mmHg}$, left ventricular pressure was $63 \mathrm{mmHg}$, left ventricular end-diastolic volume was 104\% of normal, and right ventricular end-diastolic volume $103 \%$ of normal.

Although the patient was hemodynamically stable with no pulmonary vein stenosis (PVS), a second vertical vein was incidentally noticed that originated from the proximal ligated vertical vein through the accessary hemiazygos vein, which drained into the superior vena cava (Figure 3).

On initial assessment, it appeared as though the second vertical vein originated from the same location as the first vein. However, the first vertical vein was obviously dominant; therefore, the second vessel would have had much less perfusion and would have been difficult to be identified before surgery. After surgery, it became visible as it had not spontaneously atrophied but rather had increased perfusion. At the time of discovery, it appeared that the RV volume load was tolerable and would be managed by careful followup. However, at the next check-up, tricuspid regurgitation had gradually increased. The RV volume load appeared to be worsening, and we decided to perform occlusion of the second vertical vein.

Next year of first catheter, we performed the treatment. The right femoral vein was accessed with a $5 \mathrm{Fr}$ venous sheath, and the accessary hemiazygos veins were accessed from the SVC using a $4 \mathrm{Fr} / 100 \mathrm{~cm}$ GlidecathII catheter with a Berenstein tip (Terumo). A diagnostic contrast angiography using a 2.2 Fr Progreat $\beta^{3}$ microcatheter (Terumo) was also performed in the SVC and left pulmonary with occlusion 4 Fr wedge 
in the azygos vein. The left pulmonary artery (LPA) mean pressure measured $22 \mathrm{mmHg}$, showing an increase from the previous year. We carefully performed coil embolization to avoid occlusion of the pulmonary vein. The first coil ( $8 \mathrm{~mm} \times 250 \mathrm{~mm}$ MICRUSFRAME@C coil; Johnson \& Johnson) was introduced in close proximity to the origin of the $\mathrm{VV}$ shunt to avoid occlusion of the pulmonary vein, and angiography showed it was well positioned. Additional coils (6mm x 250mm,5mm x 200mm, 4 x 150mm DELTAFILLß) ; Johnson \& Johnson) and a final one (3mm x 100mm AZURß[?] ; Terumo) were placed to ensure complete occlusion (Figure 4). Repeat angiography showed disappearance of the residual flow from the VV shunt. Mean left pulmonary artery pressure measurements revealed the same result before and after the procedure, and the patient was discharged from the hospital 2 days later.

\section{Discussion}

Double drainage of TAPVC is a rare variant of a mixed type TAPVC, which occurs when all the pulmonary veins form a confluence and then drain to both the coronary sinus and the left innominate vein. [3]. [4]

Recently however, other variants of TAPVCs with double drainage have been reported. [5]. [6]

In our 2 cases, the pulmonary veins formed a confluence and drained into the systemic venous system via two vertical veins. The first of the vertical veins drained through the innominate vein, and the second originated from the proximal side of the ascending vertical vein.

Preoperative identification of TAPVC with double drainage has important surgical implications. Although in both cases we performed echocardiography and contrast CT before the initial surgery so as not to miss the diagnosis, we did not detect this anomaly.

Because the first ascending vertical vein was more prominent and blood flowed without obstruction, the second vertical vein had less perfusion and a smaller vessel diameter. However, after the initial surgery, perfusion to the dominant vertical vein decreased, causing an increase in blood flow to the second vertical vein. Pulmonary venous obstruction, with or without confluence stenosis, is a well-known complication occurring in approximately $8-15 \%$ of patients after surgical correction of TAPVC. [7]

It was reported that with smaller left-sided chambers and a noncompliant left atrium, an un-ligated vertical vein may improve survival by preventing a pulmonary hypertensive $(\mathrm{PH})$ crisis. [8]

An un-ligated vertical vein has been reported to atrophy spontaneously.

However, if it remains patent, it may cause right cardiac failure due to left-to-right shunting. [9]

PVS did not occur after surgery in either of the cases, and on follow-up, using chest X-rays and echocardiography for diagnostic imaging, this complication was not detected. In our institution, a follow-up diagnostic catheterization for post TAPVC surgery patients is performed 1 year after the operation routinely. If this catheterization is not performed, this anomaly can go undetected. While echocardiography is sufficient for diagnosing most TAPVC cases, cardiac catheterization is essential in a mixed variety to adequately assess drainage and possible obstruction of all 4 pulmonary veins. [10]

We performed angiography and contrast $\mathrm{CT}$ again in another patient who underwent repair of a supracardiac TAPVC 10 years ago in our institution; this patient did not present with the same anomaly. The left-to-right shunting persisted, and we did not identify right heart volume overload early as was done in Case 1 . The hemodynamics in this patient however mimicked an atrial septal defect, and the right heart volume load gradually increased as he became an adult.

On the other hand, in Case 2, there was significant right heart volume overload and it caused an exacerbation of the tricuspid regurgitation, resulting in an increase of the mean pulmonary artery pressure. Coil embolization of the second vertical vein was therefore appropriate in this case.

Previously, it was recommended that the vertical vein was deliberately not operated on in order to prevent a PH crisis, with embolization with a coil or plug to be performed if the right heart volume load increased. [11], [12] 
If we could ligate vertical vein near the pulmonary vein, these results might not be occur.

To our knowledge, this is the first case of a coil embolization for a vessel that originated from the proximal ligated vertical vein and drained into the superior vena cava through an accessory hemiazygos vein-azygos vein after a TAPVC repair.

TAPVC of mixed type is often misdiagnosed if left-to-right shunting is present like in our case. This procedure proved to be safe and provided an alternative noninvasive treatment that did not involve surgery.

\section{Conclusion}

We encountered two rare cases of mixed type TAPVC with double drainage, where the second vertical vein enlarged rather than atrophied after surgery. Echocardiography is a useful diagnostic tool in the follow-up of PVS, however, contrast enhanced CT or diagnostic catheterization should be performed to exclude a second veno-venous shunt post-surgery. Cases of left-to-right shunting that is not recognized before surgery, it can be treated with catheterization as demonstrated by our case.

\section{Author contribution}

Hiroyuki Nagao: Data curation; Formal analysis; Investigation; Software; Visualization; Writing - original draft. Kenta Tominaga: Investigation; Methodology; Resources. Naoya Kamei: Investigation; Resources; Validation. Toshikatsu Tanaka: Conceptualization; Project administration; Supervision; Validation.

\section{Reference}

1) Kanter KR. Surgical repair of total anomalous pulmonary venous connection. Semin Thorac Cardiovasc Surg Pediatr Card Surg Annu. 2006;9:40-44.

2) Darling RC, Rothney WB, Craig JM. Total pulmonary venous drainage into the right side of the heart; report of 17 autopsied cases not associated with other major cardiovascular anomalies. Lab Invest 1957;6:4464.

3) Arciprete P, Mckay R, Watson GH, Hamilton DI, Wikinson JL, Arnold RM. Double connection in total anomalous pulmonary venous connection. J. Thorac. Cardiovasc. Surg. 1986; 92: 146-152.

4) Lee ML, Wang JK, Wu MH, Chu SH, Lue HC. Unusual form of total anomalous pulmonary venous connection with double drainage. Pediatr. Cardiol. 1995; 16: 301-303.

5)Tanabe S, Nakasato M, Suzuki H, Ishikawa A, Fukasawa M, Hayasaka K. A new form of total anomalous pulmonary venous connection with double drainage. Pediatr Int. 2000 ;42:369-371.

6) Krishna MR, Gnanappa GK, Mervis J, Forsey J, Ayer JG, Orr Y. Double drainage of total anomalous pulmonary venous connection: A rare variant of mixed total anomalous pulmonary venous connection. Ann Pediatr Cardiol. $2020 ; 13: 100-101$

7) van de Wal HJ, Hamilton DI, Godman MJ, Harinck E, Lacquet LK, van Oort A. Pulmonary venous obstruction following correction for total anomalous pulmonary venous drainage: a challenge. Eur J Cardiothorac Surg. 1992;6:545-549.

8)Chowdhury UK, Subramaniam KG, Joshi K, Varshney S, Kumar G, Singh R, Venugopal P. Rechanneling of total anomalous pulmonary venous connection with or without vertical vein ligation: results and guidelines for candidate selection. J Thorac Cardiovasc Surg 2007;133:1286-1294.

9) Amoozgar H, Ahmadipoor M, Amirghofran AA

Transcatheter Closure of Partially Ligated Vertical Vein after Surgical Correction of Supracardiac Total Anomalous Pulmonary Venous Connection. J Tehran Heart Cent. 2015;10:152-155

10) Chowdhury UK, Airan B, Malhotra A, Bisoi AK, Saxena A, Kothari SS, Kalaivani M, Venugopal P. Mixed total anomalous pulmonary venous connection: anatomic variations, surgical approach, techniques, 
and results. J Thorac Cardiovasc Surg. 2008;135:106-116, 116.e1-5.

11) Narula N, Wilson N, Kumar RS. Transcatheter closure of persistent unligated vertical vein after TAPVC surgery using the Amplatzer PDA device. Catheter Cardiovasc Interv. 2007;70:117-119.

12) Kobayashi D, Forbes TJ, Delius RE, Aggarwal S. Amplatzer vascular plug for transcatheter closure of persistent unligated vertical vein after repair of infracardiac total anomalous pulmonary venous connection. Catheter Cardiovasc Interv. 2012;80:192-198.

Figure Legends

Figure 1: Contrast CT before surgery repair (behind view). White arrow indicate vertical vein(common $\mathrm{PV}$ to innominate vein)

Figure 2 : Contrast CT after surgery repair (behind view). White arrow indicate second vertical vein(originated from proximal ligated vertical vein and drain into superior vena cava )

Figure 3a : Contrast CT after surgery repair (behaind view). White arrow indicate second vertical vein(originated from proximal ligated vertical vein and drain into superior vena cava)

Figure 3b : White arrow indicate accessory hemiazygos vein

Figure 3c : left pulmonary angiogram. White arrow indicate second vertical vein into superior vena cava

Figure 4: left pulmonary angiogram after coil embolization

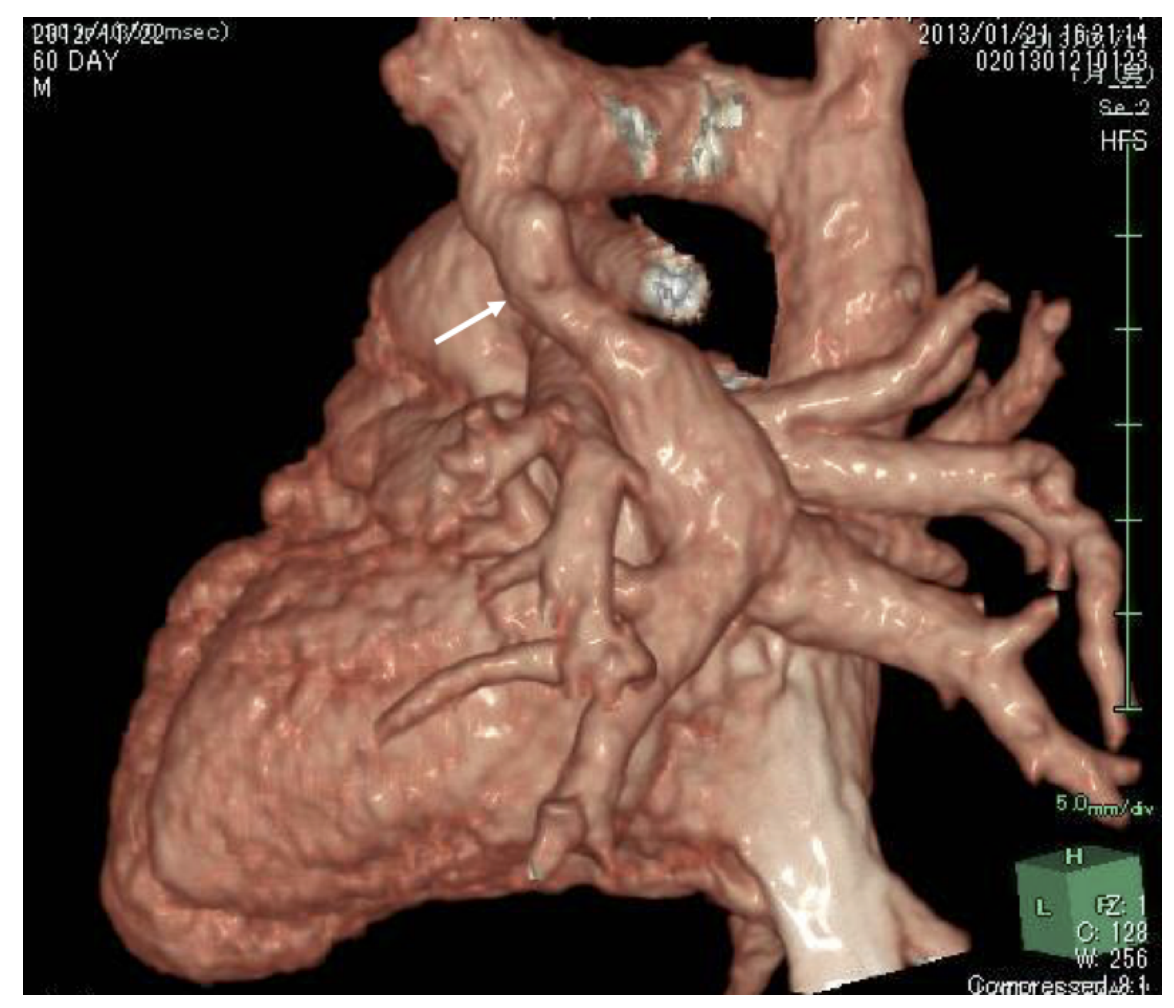




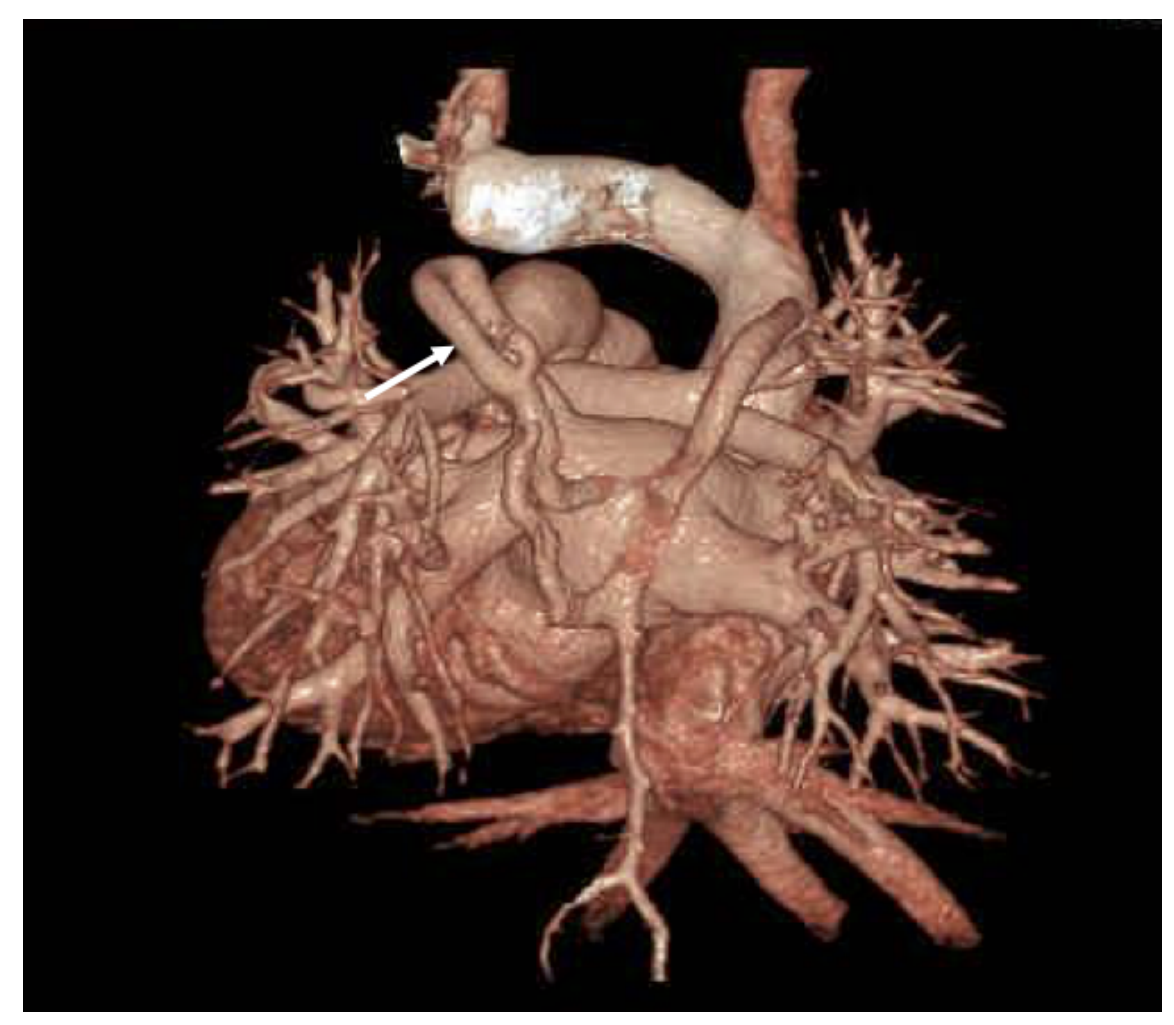




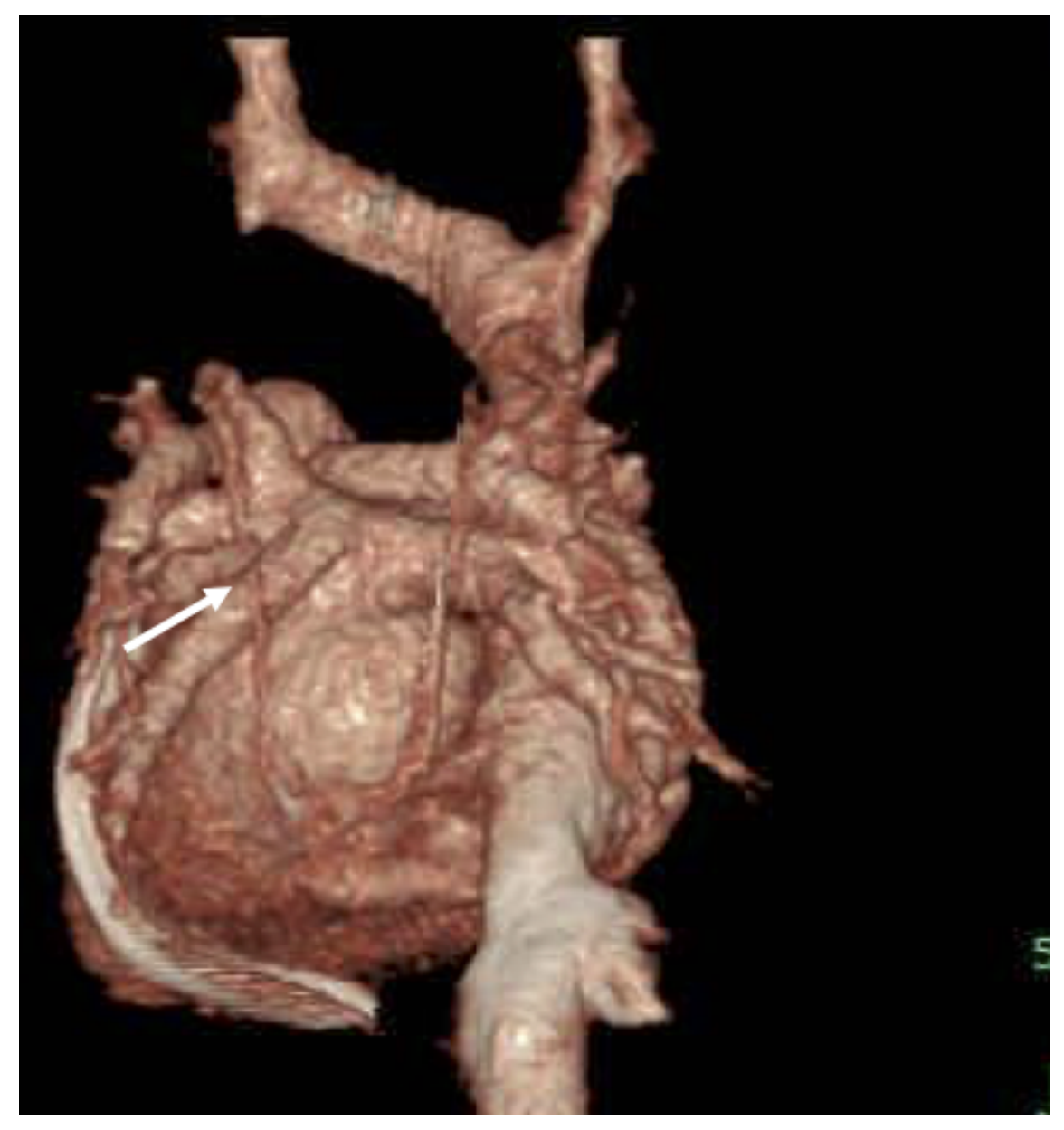




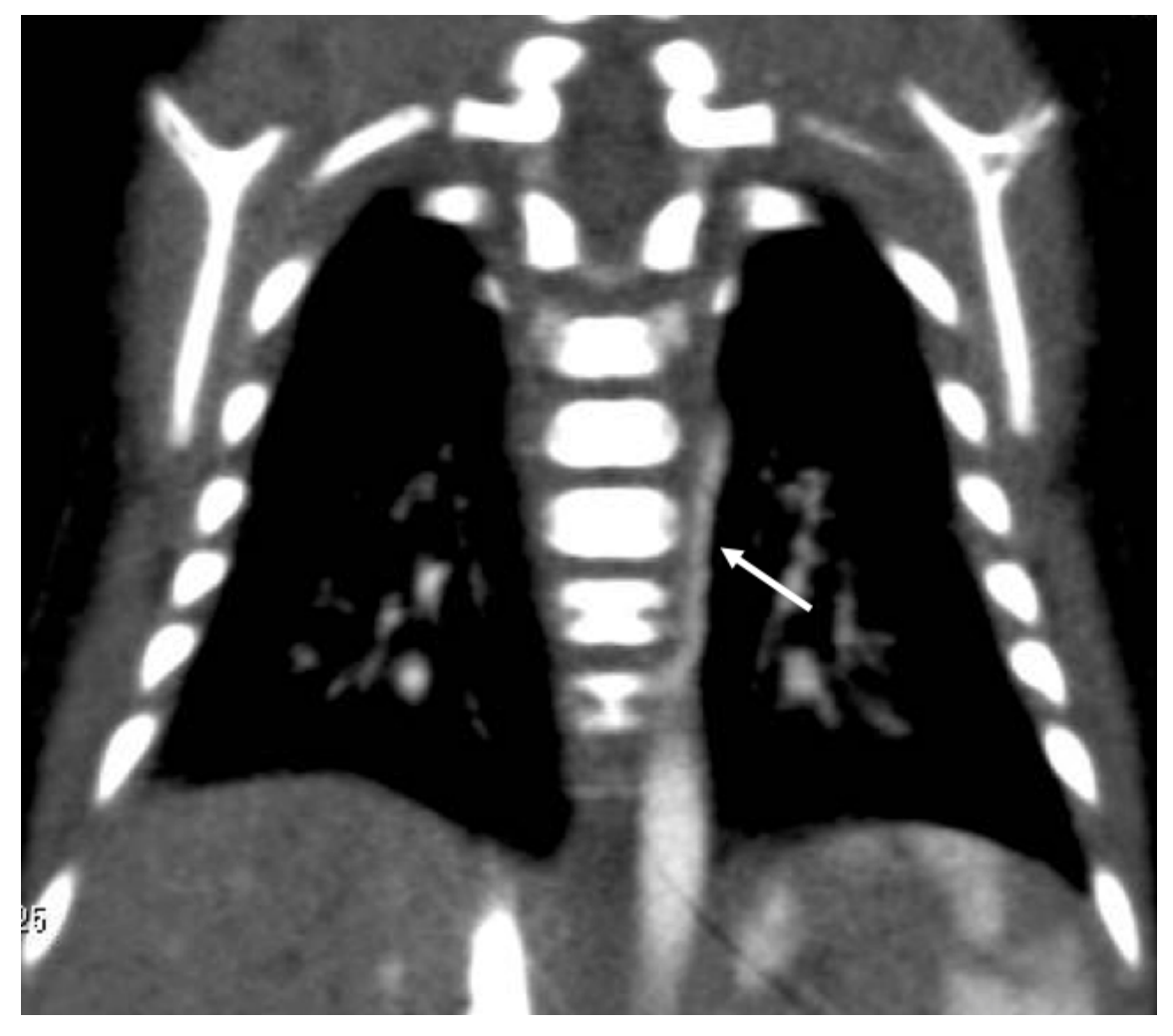




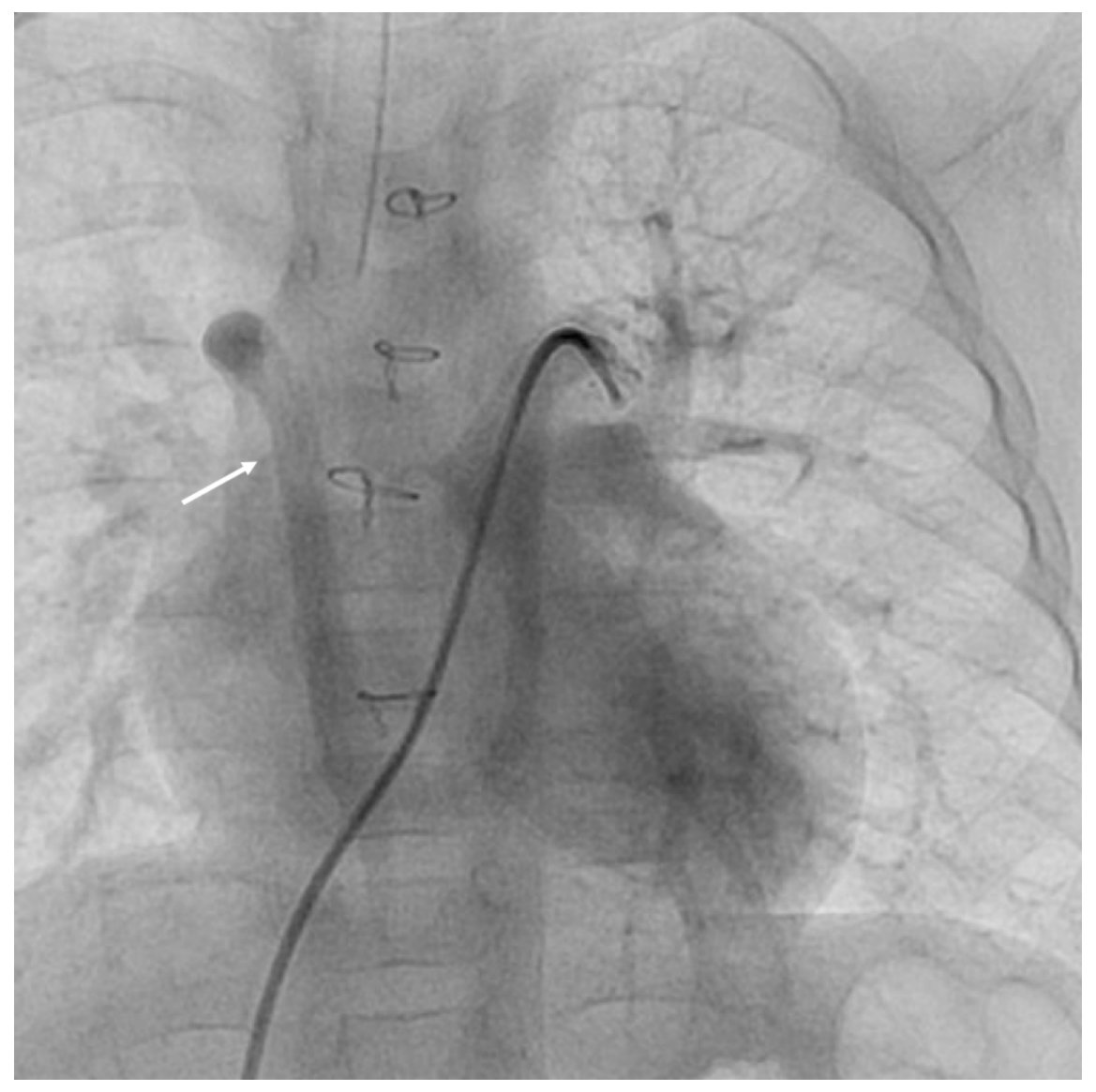




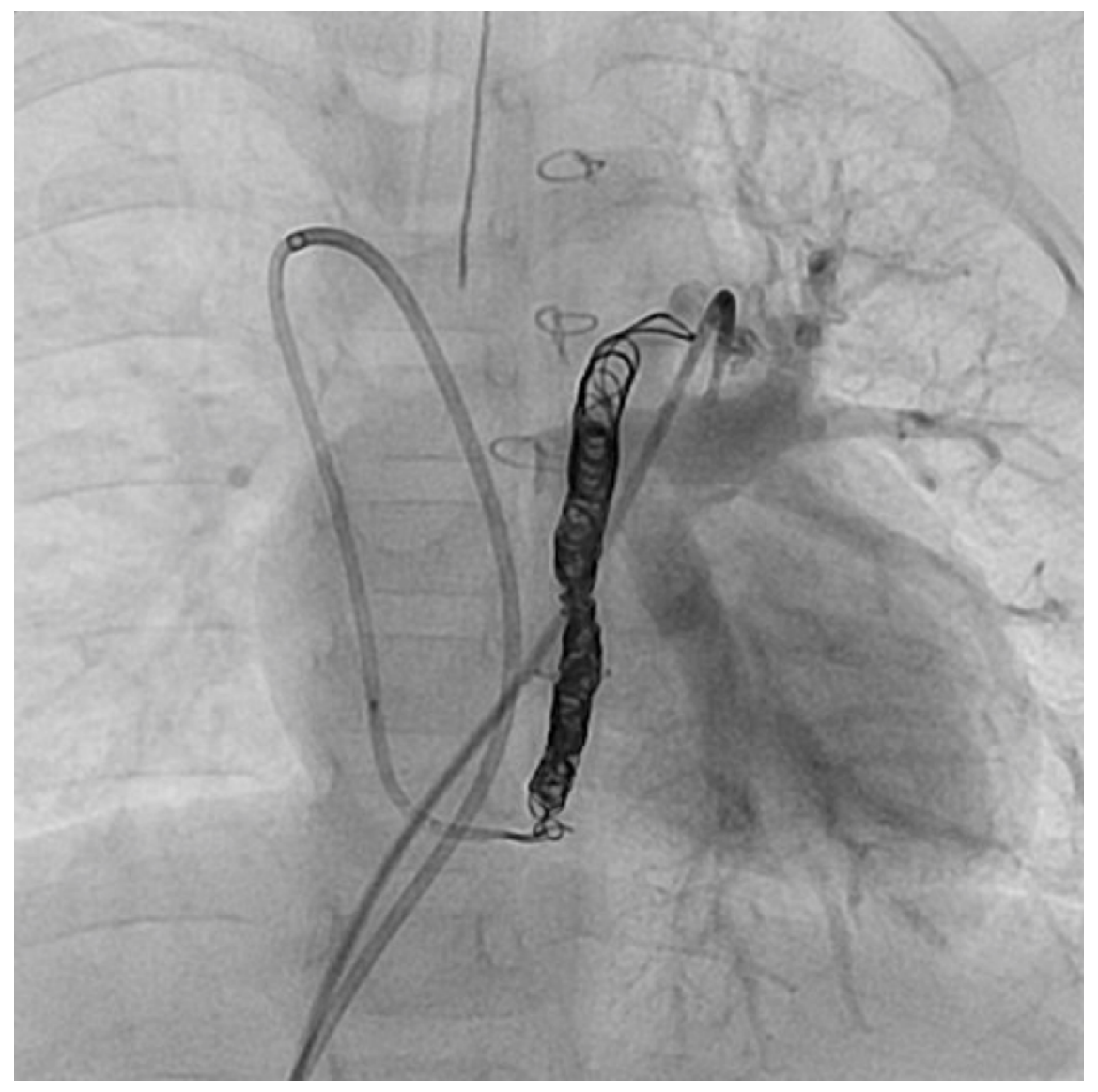

